\title{
Digitale Transformation und Open Access von Forschungsresultaten in der Schweiz
}

\author{
Dirk Verdicchio und Ingrid Kissling-Näf
}

\begin{abstract}
Zusammenfassung
Die digitale Transformation hat Einfluss auf die Arbeits- und Kommunikationsweisen der Wissenschaft und ermöglicht eine weitgehende Transparenz und Offenheit der Forschung. Die Begriffe Open Science und Open Access stehen für diese Öffnung wissenschaftlicher Prozesse und die Zugänglichmachung von Forschungsergebnissen. Für Open Access - dem freien Zugang zu wissenschaftlichen Publikationen - existieren schweizerische und internationale Programme und Strategien, die von Institutionen wie dem SNF und swissuniversities getragen werden. Neben einem Überblick über die zentralen Begriffe und Konzepte geht der Beitrag auf diese Strategien ein. Besondere Beachtung finden die Situation in Europa und der Schweiz sowie der Wandel der Geschäftsmodelle und der Publikationslandschaft.
\end{abstract}

\subsection{Was wollen Open Science und Open Access?}

Die digitale Revolution hat die Arbeitsweisen in den Wissenschaften grundsätzlich revolutioniert und das Teilen von Daten und Informationen in großem Ausmaß möglich gemacht. Sie hat damit nicht nur das Selbstverständnis der Wissenschaft verändert, sondern auch die Formen der Kollaboration und der Publikationen weltweit verändert. Eine

D. Verdicchio $(\bowtie)$

Digitale Dienste \& Open Science, Universitätsbibliothek Bern, Bern, Schweiz

E-Mail: dirk.verdicchio@ub.unibe.ch

I. Kissling-Näf

Departement Wirtschaft, Berner Fachhochschule, Bern, Schweiz

E-Mail: ingrid.kissling@bfh.ch

J. Schellinger et al. (Hrsg.), Digitale Transformation und Unternehmensführung,

https://doi.org/10.1007/978-3-658-26960-9_2 
neue Offenheit findet ihren Niederschlag in Begriffen wie Open Science, Open Access und Open Research Data. Open Science steht für den Grundgedanken, den gesamten wissenschaftlichen Prozess möglichst offen und transparent zu gestalten, um wissenschaftliche Forschung möglichst frei zugänglich, nachnutzbar und reproduzierbar zu machen. Das schließt die Beteiligung von Bürgern unter dem Label Citizen Science mit ein. Entsprechend definiert das europäische Projekt FOSTER Open Science wie folgt:

Open Science is the practice of science in such a way that others can collaborate and contribute, where research data, lab notes and other research processes are freely available, under terms that enable reuse, redistribution and reproduction of the research and its underlying data and methods. ${ }^{1}$

Der EU Open Science Monitor gibt einen anschaulichen Überblick über die Charakteristika und Indikatoren von Open Science (Abb. 2.1). Zentral sind hier das Teilen der Forschungsdaten (Open Research Data), die Transparenz des Wissenschaftskommunikationsprozesses (Open Scholarly Communication) sowie der offene Zugang zu wissenschaftlichen Publikationen (Open Access to Publications). Diese Öffnung bedingt strukturelle, kulturelle und praktische Justierungen des wissenschaftlichen Prozesses. Dabei muss betont werden, dass der Begriff der Offenheit weder theoretisch noch praktisch klar umrissen ist und kontextuell interpretiert wird und werden muss (siehe Reichmann 2017; Levin und Leonelli 2017). Illustrieren lässt sich das an den Diskussionen zu Open Research Data und FAIR Data. Die FAIR-Prinzipien, entworfen von FORCE 11, formulieren Findbarkeit, Zugänglichkeit, Interoperabilität und Nachnutzbarkeit von Forschungsdaten als zentrale Momente des Umgangs mit Forschungsdaten. ${ }^{2}$ Das Konzept sieht die Offenheit der Informationen über Forschungsdaten vor, aber nicht zwingend die Offenheit der Forschungsdaten selbst, die sich gerade im Bereich der sensiblen Forschungsdaten nur mit großen Einschränkungen umsetzen lässt.

Open Science verspricht Vorteile im Bereich der Effizienz wissenschaftlicher Forschung, da eine Vielzahl von Ergebnissen für weitere Forschungstätigkeiten genutzt und Duplizierungen von Forschungsergebnissen verhindert werden können. Kollaborationen werden durch Open Science ebenso begünstigt wie die Demokratisierung wissenschaftlichen Wissens, wie es in den Diskursen zu Citizen Science entworfen wird (vgl. Arza und Fressoli 2017). Darüber hinaus verschaffen offene Praktiken Forschenden eine höhere Visibilität und mehr Zitationen (McKiernan et al. 2016). Eine von der Europäischen Kommission in Auftrag gegebene Studie zu den wirtschaftlichen Effekten von FAIR Data kommt $2018 \mathrm{zu}$ dem Schluss, dass ein Verzicht auf das Teilen von Forschungsdaten 10,2 Mrd. EUR jährlich kosten würde und formuliert entsprechende Empfehlungen zur Förderung von Infrastrukturen und Praktiken für Forschungsdaten (European Commission 2018). Bei der Betrachtung der entsprechenden Literatur bleiben - trotz der momentan

\footnotetext{
${ }^{1}$ https://www.fosteropenscience.eu/foster-taxonomy/open-science-definition.

${ }^{2} \mathrm{https} / / / \mathrm{www}$. force11.org/group/fairgroup/fairprinciples.
} 


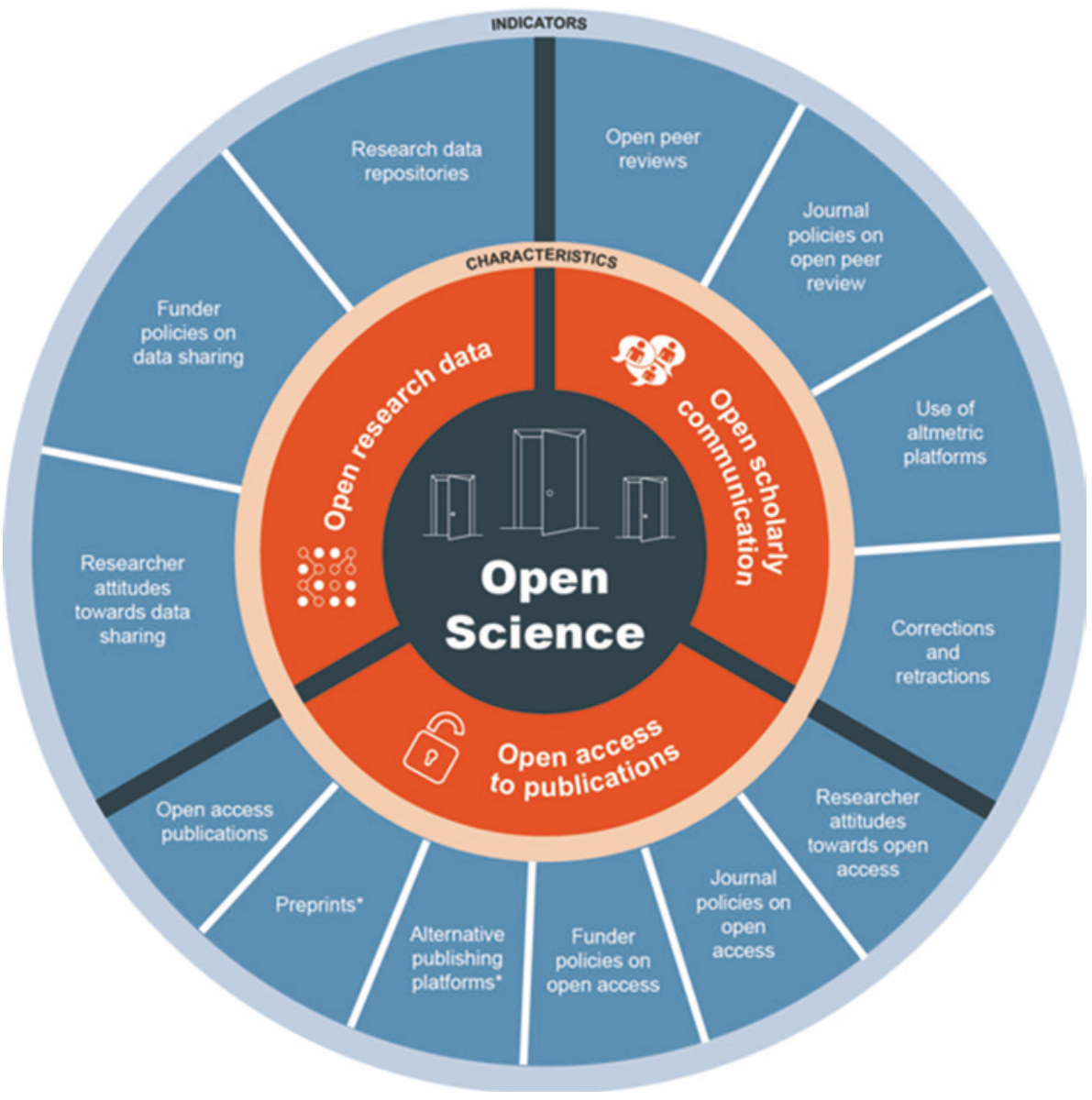

Abb. 2.1 Foster Open Science „Wheel“. (European Commission 2017, S. 6)

noch bestehenden begrifflichen und konzeptuellen Unklarheiten - kaum Zweifel, dass Open Science allen an der Wissenschaft direkt und indirekt Beteiligten zugutekommt. Eine Ausnahme bilden hier vielleicht diejenigen, die von der Regulierung des Zugangs zu wissenschaftlichem Wissen profitieren.

Open Access, der kostenfreie Zugang zu wissenschaftlicher Literatur über das Internet, ist ein wichtiger Baustein von Open Science und blickt auf eine nahezu 30-jährige Geschichte zurück. Die primären Ziele von Open Access sind eng mit den Möglichkeiten der Verbreitung von Informationen über das Internet verknüpft. Die Anfänge von Open Access lassen sich auf die Einrichtung des Servers arXiv durch Alan Ginsparg im Jahr 1991 zurückführen, der die Verbreitung von physikalischen Preprints, die bis dahin per Post versandt wurden, deutlich beschleunigte. Durch arXiv und andere Preprint-Server wurde der Grundstein für den sogenannten grünen Weg gelegt, wie die Zugänglichmachung 
von Texten über Repositorien bezeichnet wird. Ein weiterer technischer Meilenstein war die Gründung der Open Archive Initiative und das im Jahr 2000 entwickelte Open Archive Initiative Protocol for Metadata Harvesting (OAI-PMH), welches das Harvesten (automatisiertes Sammeln) von Daten aus Repositorien erlaubt und die Etablierung von Open-Access-Repositorien förderte (siehe dazu Deppe und Beucke 2017).

In den Jahren 2001 und 2003 fanden in Budapest, Bethesda und Berlin Konferenzen statt, die als Auftakt der Open-Access-Bewegung gesehen werden können. Jede dieser drei Konferenzen mündete in eine Deklaration ${ }^{3}$, die den Open-Access-Gedanken genauer präzisiert. Die Berliner Deklaration von 2003, die in den folgenden Jahren auch vom Schweizerischen Nationalfonds SNF, der Rektorenkonferenzen der Fachhochschulen und der Universitäten, den Akademien der Wissenschaften und den meisten schweizerischen Universitäten unterzeichnet wurde, formuliert das Ziel, wissenschaftliche Informationen weltweit über das Internet frei zugänglich zu machen. Dabei soll allen Nutzenden das Recht zugestanden werden, diese Informationen ,zu kopieren, zu nutzen, zu verbreiten, zu übertragen und öffentlich wiederzugeben sowie Bearbeitungen davon zu erstellen und zu verbreiten, sofern die Urheberschaft korrekt angegeben wird“. Darüber hinaus macht die Berliner Erklärung deutlich, dass Open Access nicht nur auf die Zugänglichmachung von textuellen Publikationen zielt, sondern auch weitere wissenschaftliche Informationen frei zugänglich gemacht werden sollen (siehe Berliner Erklärung ${ }^{4}$ ). Der Umstand, dass die Berliner Erklärung weltweit von über 500 Institutionen unterzeichnet wurde, macht diese Deklaration zu einem zentralen Dokument der Open-Access-Bewegung.

Im Zuge der internationalen Aktivitäten rund um Open Access haben sich folgende Formen und Begriffe etabliert: Grün Open Access wird die Selbstarchivierung, also die Zweitveröffentlichung von wissenschaftlichen Texten in einem Repositorium genannt. Solche Open-Access-Repositorien, wie bspw. BORIS an der Universität Bern, werden in der Regel von Institutionen getragen. Wenn die Erstveröffentlichung bei einem Verlag erfolgte und eine Übertragung der Verbreitungsrechte an den Verlag (copyright transfer agreement) stattgefunden hat, dann bestimmt der Verlag darüber, welche Version des Texts $^{5}$ publiziert werden und mit was für einem Embargo die Zweitveröffentlichung erfolgen darf. Die Erstveröffentlichung von Publikationen in einer Open-Access-Zeitschrift oder als Open-Access-Buch wird Gold Open Access genannt. Die Publikation ist hier mit dem Erscheinen frei zugänglich und ist mit einer Lizenz ausgestattet, die

\footnotetext{
${ }^{3}$ Budapest Open Access Initiative (2002), Bethesda Statement On Open Access Publishing (2003) und die Berlin Declaration on Open Access to Knowledge in the Sciences and Humanities (2003). ${ }^{4}$ https://openaccess.mpg.de/Berlin-Declaration.

${ }^{5}$ In der Regel erlauben die Verlage die Publikation der begutachteten Manuskriptversion (auch post-print oder author accepted manuscript genannt).
} 
die legitime Form der Nachnutzung determiniert. ${ }^{6}$ Einige Open-Access-Zeitschriften verlangen Publikationsgebühren (sogenannte article processing charges oder APCs), andere Zeitschriften finden Wege der Finanzierung, die ohne Zahlungen durch Autoren auskommen (s. u.). Zeitschriften, die keine Publikationsgebühren erheben, werden manchmal auch Diamond-Open-Access-Zeitschriften genannt. Die dritte Form der Umsetzung des Open-Access-Gedankens nennt man Hybrid Open Access. Hier werden Zeitschriftenartikel in klassischen Subskriptionszeitschriften publiziert und gegen Gebühr frei zugänglich gemacht. Hybrid Open Access wird häufig als transitorisches Modell gesehen, das Zeitschriften dabei helfen soll, den geordneten Übergang zu einem Gold-Open-Access-Modell zu bestreiten, indem das Geschäftsmodell nach und nach umgestellt wird. Bisher gibt es jedoch keine Anzeichen dafür, dass dies im großen Stil erfolgen wird. ${ }^{7}$

Neben dem offenen Zugang und der damit verbundenen besseren Verfügbarkeit wissenschaftlichen Wissens, lässt sich durch Open Access die Kommunikation wesentlich beschleunigen und prinzipiell günstiger gestalten. Diesen Zielen stehen die historisch gewachsenen Publikations- und Verbreitungsformen entgegen. Insbesondere das Subskriptionsmodell für Zeitschriften und die damit verbundenen Paywalls unterbinden und beschränken den Zugang zu den Forschungsergebnissen, sie stellen damit ein Hindernis für die wissenschaftliche Forschung und die gesellschaftliche Aneignung wissenschaftlichen Wissens dar und stehen damit dem Grundsatz entgegen, dass wissenschaftliches Wissen für alle zugänglich sein soll (vgl. z. B. Merton 1973).

Die Zugänglichkeit wird zusätzlich durch starke Anstiege der Subskriptionskosten erschwert, die dazu führen, dass Bibliotheken Zeitschriftensubskriptionen abbestellen und Anschaffungen reduzieren müssen. Der Umstand, dass sich auch die Harvard University 2012 gezwungen sah, Zeitschriftenabonnemente zu kündigen, kann als Indiz dafür gesehen werden, dass heute keine Universität ihren Forschenden eine vollständige Versorgung mit wissenschaftlichen Informationen garantieren kann und stellt die ökonomische und wissenschaftliche Nachhaltigkeit der Subskriptionsmodelle insgesamt infrage (Sample 2012).

Das traditionelle Publikationsmodell steht aber auch deswegen in der Kritik, weil der überwiegende Teil wissenschaftlicher Forschung öffentlich finanziert ist, die Ergebnisse der Öffentlichkeit jedoch nicht zur Verfügung stehen. Vielmehr muss der Zugang zu diesem Wissen abermals mit öffentlichen Geldern bezahlt werden. Die Frage nach der Gerechtigkeit eines solchen Modells stellt sich gerade wegen der außerordentlich hohen Gewinne, die einige Verlage mit der Verbreitung öffentlich finanzierter Forschungsergebnisse erzielen, wobei sie Profitraten von bis zu über $40 \%$ erreichen (Siehe z. B. Morrison 2012, S. 11 ff., 2014; Buranyi 2017).

\footnotetext{
${ }^{6}$ Meist handelt es sich dabei um Creative-Commons-Lizenzen (siehe https://creativecommons. org/).

${ }^{7}$ Zu Hybrid Open Access in der Schweiz am Beispiel der ETH Zürich siehe Christen 2017.
} 
Zu den Eigenheiten des wissenschaftlichen Publikationssystems gehört, dass die Verlage weder für die Manuskripte, noch für die redaktionellen Tätigkeiten oder die Qualitätssicherung über Peer Review zahlen, die von Forschenden entweder im Rahmen ihrer Arbeitszeit oder ihrer Freizeit getätigt werden. Dafür treten die Autoren die exklusiven Verbreitungsrechte an ihren Werken an die Verlage ab, die die Verbreitung dieser Werke beliebig monetarisieren und kontrollieren können. So kann es vorkommen, dass die Urheber für die Nachnutzung ihrer eigenen Texte, die sie dem Verlag kostenlos überlassen haben, abermals eine Gebühr bezahlen müssen.

\subsection{Open-Access-Politiken in Europa}

Seit 2012 gibt es in Großbritannien, den Niederlanden und Österreich national koordinierte Pläne zu Open Access ${ }^{8}$, die in der Schweiz als Anlass und Vorbild zum Entwurf einer Nationalen Open-Access-Strategie dienten, die 2017 verabschiedet wurde (s. u.). Für Schweizer Forschende war aber bereits die Open-Access-Politik der Europäischen Union direkt relevant. Das siebte Forschungsrahmenprogramm (FP7) der Europäischen Kommission (2007-2013) empfahl erstmals die Open-Access-Publikation der Ergebnisse der geförderten Forschung. Darüber hinaus wurde ein ,post grant open access fund" eingerichtet, über den sogenannte Article Processing Charges (APC), d. h. Gebühren für Publikationen abgeschlossener FP7-Projekte beantragen konnten. ${ }^{9}$

Im Rahmen von FP7 wurde 2010 das Projekt „Open Access Infrastructure for Research in Europe“ (OpenAire) ins Leben gerufen, das die Zugänglichkeit der Publikationen und Daten sicherstellen soll, die im Rahmen des Programms entstanden sind. $\mathrm{Zu}$ den Infrastrukturen, die hierfür geschaffen wurden, gehört auch das Repositorium Zenodo, das am CERN betrieben wird und für alle Forschenden, unabhängig von der institutionellen Zugehörigkeit, zur Verfügung steht (Manola et al. 2015). Im Jahr 2014 folgte das achte Forschungsrahmenprogramm, das den Titel Horizon 2020 trägt und Open Access mandatiert. Publikationen aus dem Bereich der sogenannten MINT-Fächer müssen spätestens sechs Monate nach Erscheinen Open Access verfügbar gemacht werden und Publikationen aus dem Bereich der Geistes- und Sozialwissenschaften nach spätestens 12 Monaten. Außerdem wird erwartet, dass für jedes Projekt ein Forschungsdatenplan erstellt wird und die Forschungsdaten publiziert werden. Eine wichtige Neuerung von Horizon 2020 ist die Compliance-Überwachung. Wie bei vielen Forschungsförderern und Institutionen, hatte das Open-Access-Mandat bei EU-Projekten bis dahin eher den Charakter einer Empfehlung. Dies änderte sich 2018 als der European

\footnotetext{
${ }^{8}$ Siehe dazu https://ukrio.org/open-access-research-report-of-the-finch-group/, https://www.openaccess.nl/, https://www.oana.at.

${ }^{9} \mathrm{https}: / /$ ec.europa.eu/research/science-society/document_library/pdf_06/open-access-pilot_en.pdf, https://ec.europa.eu/digital-single-market/en/news/results-fp7-post-grant-open-access-pilot.
} 
Research Council (ERC) Universitäten aufforderte, der in der Fördervereinbarung festgehaltenen Verpflichtung zu Open Access nachzukommen.

Im September 2018 hat cOAlition S, eine Allianz von 16 Forschungsförderern, dem ERC und der Europäischen Kommission Open-Access-Richtlinien unter dem Namen Plan S veröffentlicht (cOAlition S 2018; siehe auch Tulney 2018). Die Richtlinien schlagen eine deutliche Verschärfung der Open-Access-Auflagen vor und zielen auf eine Transformation des Publikationswesens. $\mathrm{Zu}$ den zehn Maßnahmen, die Plan S vorsieht, gehören der Verzicht auf die Übertragung der Verbreitungsrechte an die Verlage, die Verpflichtung zur Nutzung einer Lizenz, die der Creative-Commons-Attribution entspricht (CC BY), die Festsetzung einer maximalen Höhe für Publikationsgebühren und Sanktionen bei Nichtbefolgung des Open-Access-Mandats. Mit dem Grundsatz, dass Publikationen in hybriden Open-Access-Zeitschriften nicht akzeptiert werden, soll die Transformation von Subskriptionszeitschriften in Open-Access-Zeitschriften stimuliert werden. Diese Grundsätze sollen für alle geförderten Publikationen gelten, Buchpublikationen eingeschlossen. Plan S hat weltweit Reaktionen provoziert. Der Aufruf zu Rückmeldungen und Kritik des Plans führte zu ca. 600 Eingaben. Während die Gegner einer Verpflichtung zu Open Access den Plan grundsätzlich ablehnen, wurde er von Befürwortern von Open Access zwar prinzipiell begrüßt aber teilweise als zu wenig differenziert und schlecht umsetzbar kritisiert. Dennoch adressiert der Plan S wichtige Punkte, die die Durchsetzung von Open Access behindern und hat daher das Potenzial, die Publikationslandschaft in Richtung Open Access zu verändern.

\subsection{Open-Access-Politik in der Schweiz}

Wie Abb. 2.2 zeigt, führt die Schweiz erstaunlicherweise mit $39 \%$ Open-Access-Publikationen weltweit die Länderliste im Vergleich an. Durchschnittlich sind nur $30 \%$ der Publikationen auf dem goldenen und grünen Weg frei zugänglich, ${ }^{10}$ siehe auch Gutknecht et al. 2016.

Lange Zeit wurde Open Access in der Schweiz eher zurückhaltend verfolgt. Obwohl der Schweizerische Nationalfond (SNF) 2008 eine Verpflichtung zur Selbstarchivierung von Artikeln einführte, wurde die Einhaltung der Vorgabe nicht überwacht. Erst 2013 hat der SNF eine eigentliche Open-Access-Politik postuliert und die Publikationspolitik mit Richtlinien, für die mit öffentlichen Geldern finanzierten Forschungsresultate versehen. Dabei setzte der SNF bewusst auf die Förderung des goldenen Wegs. Article Processing Charges (APCs) für die Publikation von Artikeln in Open Access wurden mit einem Betrag von CHF 3000 unterstützt. Im Jahr 2014 wurde dann die Open-Access-Politik auf Buchpublikationen ausgeweitet. Gefördert werden seitdem nur noch digitale Versionen von Büchern (Produktionskosten). Darüber hinaus müssen Buchpublikationen spätestens 24 Monaten nach deren Erscheinen auf einem Repositorium zugänglich gemacht werden.

\footnotetext{
${ }^{10} \mathrm{https}: / /$ ec.europa.eu/info/research-and-innovation/strategy/goals-research-and-innovation-policy/ open-science/open-science-monitor/trends-open-access-publications_en.
} 


\section{Percentage of Open Access publications (Gold and Green) by country}

Source: Consortium's own analysis of Scopus and Unpaywall databases - Reference date: 20092017

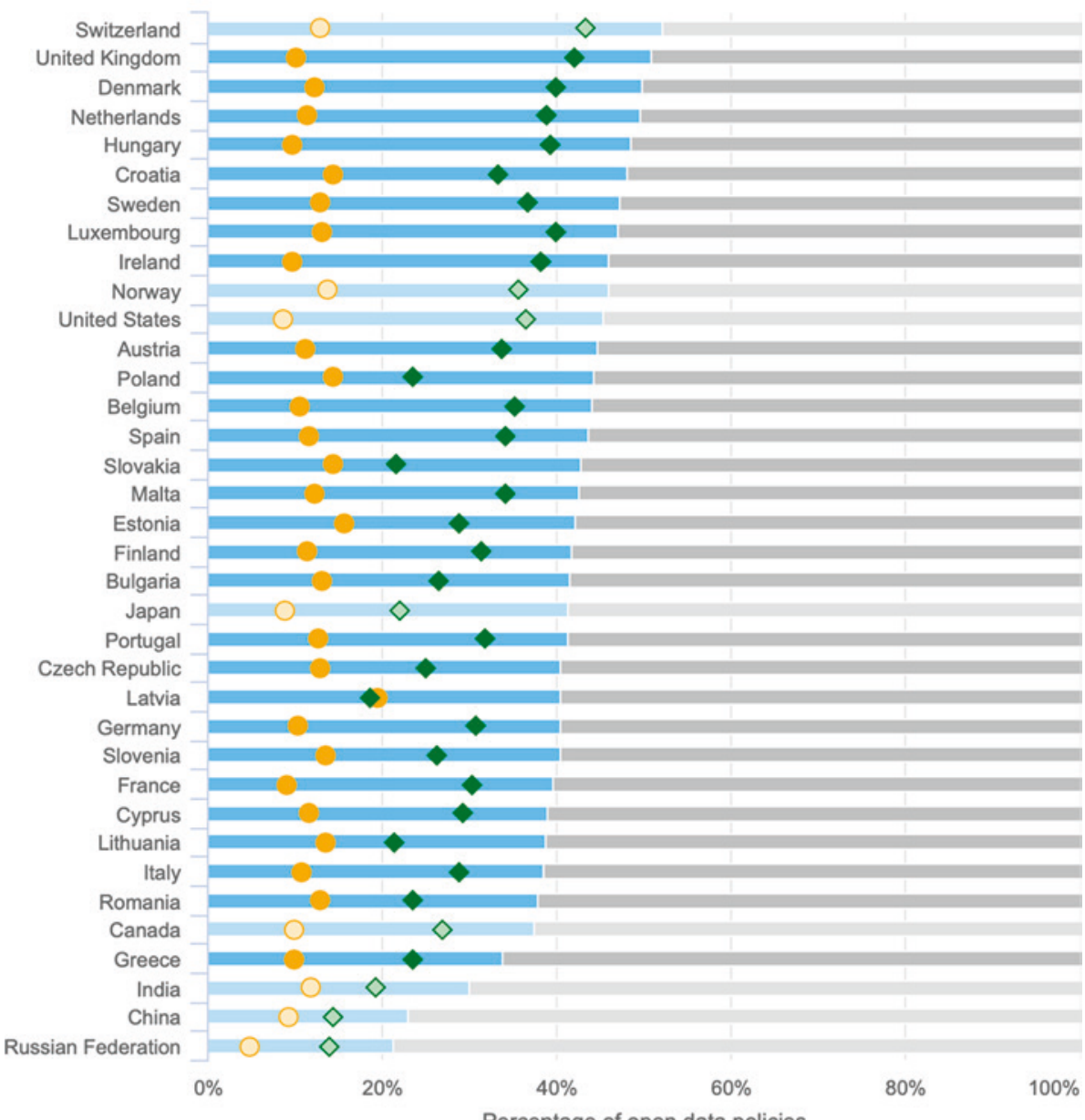

Percentage of open data policies
Green OA
Gold OA
OA
Not OA

Abb. 2.2 EU Open Science Monitor. (European Commission 2019) 


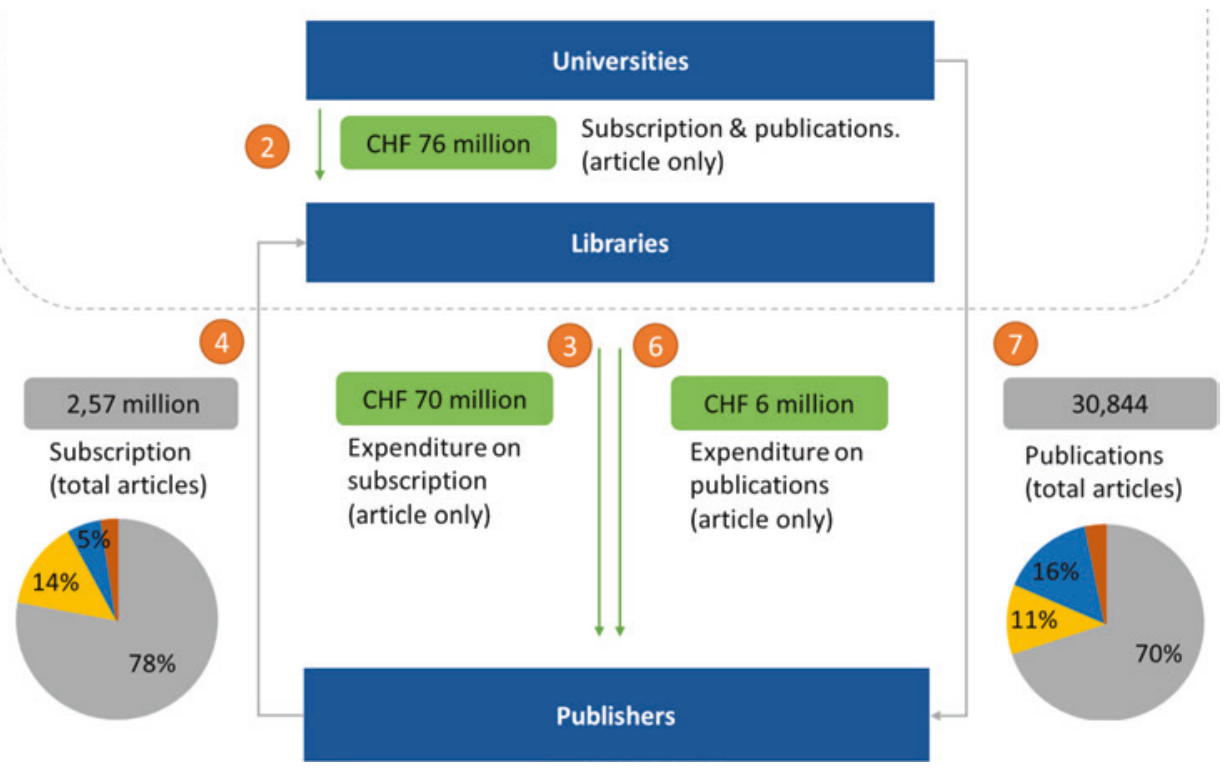

Abb. 2.3 Finanzflüsse im Publikationswesen der Schweiz im Jahr 2015. (CEPA 2017)

Die Richtlinien des SNF erzeugten einen Sturm der Entrüstung, ${ }^{11}$ die Schweizer Verlagshäuser sammelten Unterschriften gegen die Publikationspolitik des SNF ${ }^{12}$ und im Parlament wurde eine Interpellation eingereicht, weil das Schweizer Verlagswesen durch Open Access bedroht sei. ${ }^{13}$ In der Folge fanden im Frühsommer 2014 erste Gespräche zwischen dem SNF und den Verlegern statt, als Kompromiss einigte man sich auf ein Pilotprojekt mit Schweizer und deutschen Verlegern in den Geistes- und Sozialwissenschaften. Das Projekt OAPEN-CH sollte in Anlehnung an Studien, die in den Niederlanden (OAPEN-NL) und Großbritannien (OAPEN-UK) durchgeführt wurden, untersuchen, welche Auswirkungen die Publikation von Büchern in Open Access auf den Verkauf der gedruckten Ausgaben haben. Das Projekt sollte Daten über Kosten und Verkäufe liefern, aber auch einen gemeinsamen Lernprozess von Verlegern, Autoren und Bibliotheken initiieren. Speziell interessierten die Effekte des grünen und goldenen Wegs für die Visibilität und den Absatz von Büchern. In diesem Sinne wurde ein experimentelles Design gewählt, das Open-Access-Bücher mit gedruckten Monografien bezüglich Zitation, Kosten, Absatz etc. verglich. Die Ergebnisse bestätigten die Befunde der Vorgängerstudien in den Niederlanden und Großbritannien. So stellt der Schlussbericht fest, dass

\footnotetext{
${ }^{11}$ Siehe: Weik (2014), Hirschi (2014), Hagner (2014).

${ }^{12}$ „Fonds national suisse de la recherche scientifique FNS-SNSF: L'édition académique en danger! Die akademischen Verlage sind in Gefahr!“.

${ }^{13}$ Savary (2014).
} 
(e)ine digitale, übers Internet frei verfügbare Ausgabe ... die Auffindbarkeit, Sichtbarkeit und Nutzung von Monographien (erhöht). Open Access wirkt sich nicht negativ auf den Verkauf des gedruckten Buches aus. Fazit: Für die akademische Gemeinschaft und die Gesellschaft insgesamt sowie für Verleger und Geldgeber ist es von Vorteil, Open Access für Monographien zu ermöglichen und zu unterstützen (Ferwerda et al. 2018, S. 3).

Ende 2015 beauftragte das Staatssekretariat für Bildung, Forschung und Innovation (SBFI) swissuniversities, gemeinsam mit dem SNF eine nationale Strategie auszuarbeiten. Konkreter Handlungsbedarf wurde darin gesehen, Open Access als übergeordnetes Ziel zu fördern, Kostentransparenz bei öffentlichen Ausgaben zu schaffen und die Aktivitäten der Stakeholder einschließlich der Bibliotheken zu koordinieren (SBFI 2015). Damit war der Grundstein für eine schweizweite Koordination der OpenAccess-Aktivitäten zwischen SNF, Hochschulen, Förderprogrammen und Bibliotheken gelegt. In der Folge wurde eine sogenannte Finanzflussanalyse erarbeitet. Diese wies aus, dass Schweizer Bibliotheken 2015 rund 70 Mio. an Subskriptionenkosten ausgaben, damit Artikel gelesen werden konnten. Die Erhebungen zeigten zudem, dass sich die Kosten der Bibliotheken für Gold Open Access und hybride Publikationen auf rund sechs Millionen Franken beliefen. Zwei Millionen Franken flossen zum damaligen Zeitpunkt in Infrastrukturen für Open Access. Etwa $11 \%$ der Schweizer Artikel wurden Open Access veröffentlicht, $16 \%$ standen auf dem grünen Weg zur Verfügung und $3 \%$ waren hybride Artikel (siehe Abb. 2.3).

Die nationale Strategie Open Access wurde Ende 2017 von der Plenarversammlung von swissuniversities verabschiedet. Sie hält als Zielsetzung fest:

Bis 2024 sollte wissenschaftliches Publizieren in der Schweiz OA sein, alle mit öffentlichen Geldern finanzierten wissenschaftlichen Publikationen müssen im Internet frei zugänglich sein. Die OA-Landschaft wird aus verschiedenen OA-Modellen bestehen (swissuniversities und Schweizerischer Nationalfonds 2016).

Die Strategie betont die Autonomie der Hochschulen und setzt auf eine weitgehende Flexibilität der Umsetzung von Maßnahmen, um das Ziel zu erreichen. Dennoch sollen die Maßnahmen, die in einem Aktionsplan konkretisiert wurden, koordiniert werden: So sollen die Open-Access-Politiken der verschiedenen Akteure abgestimmt werden. Eine Verhandlungsdelegation wurde gegründet, die mit Verlagen Read-and-Publish-Verträge aushandelt. Es sollen, wenn es sinnvoll und möglich ist, Ressourcen gebündelt werden. Und es sollen Alternativen zu den gängigen Publikationsformen geschaffen und unterstützt werden. Darüber hinaus werden Kommunikations- und Sensibilisierungsmaßnahmen ergriffen und ein Monitoring der Umsetzung soll erfolgen. Eine besondere Bedeutung kommt der Reform der Forschungsevaluation zu, die für die Forschenden die Anreize zur Publikation in Open Access schaffen soll. Die Strategie betont zudem die Wichtigkeit eines Zweitveröffentlichungsrechts, das es den Verfassern von wissenschaftlichen Publikationen erlaubt, ihre Publikationen unabhängig von der Einwilligung eines Verlags über den grünen Weg Open Access zugänglich zu machen. 
Der SNF nimmt für die Umsetzung der Open-Access-Politik im Verbund mit den Hochschulen eine Vorreiterrolle ein und strebt an, dass alle von ihm geförderte Forschungsergebnisse bis 2020 frei zugänglich sein sollen. Entsprechend wurden 2018 die Fördermaßnahmen angepasst. So wurde der maximale Förderbetrag für APCs aufgehoben und die Publikationsunterstützung von den Anträgen für Projektbeiträge entkoppelt. Forschende können nun unabhängig von der Laufzeit ihrer Projekte Mittel für Open-Access-Publikationen beantragen. Zudem wurde das Embargo für die Zugänglichmachung von Büchern auf 12 Monate verkürzt und es wurde ein Compliance-Monitoring eingeführt. ${ }^{14}$

\subsection{Geschäftsmodelle und der Wandel der Publikationslandschaft}

Der Publikationsmarkt war in den letzten Jahrzehnten von kommerziellen Verlagen wie Elsevier, Springer und Wiley dominiert. Elsevier z. B. gibt rund 2500 Zeitschriften heraus. Das Oligopol am Publikationsmarkt erschwert zusammen mit den bisher üblichen Non-Disclosure-Agreements, die eine Kommunikation der Lizenzbedingungen und Preise untersagen, die Transparenz der Publikations- und Subskriptionskosten (siehe z. B. Larivière et al. 2015). Diese Konstellation hat zur Folge, dass die Bibliotheken in einer schlechten Verhandlungsposition sind und finanziell zunehmend unter Druck geraten.

Der technologische Wandel und der wachsende Zuspruch, den Open Access und Open Science finden, verändert aber auch die Publikationslandschaft. So erleben kommerzielle Start-ups und nichtkommerzielle Initiativen im Bereich alternativer Metriken, alternativer Publikationsformen und Repositorien eine Blüte. Große Verlage versuchen dagegen das tradierte Finanzierungsmodell mit den ertragreichen Closed Journals so lange wie möglich am Leben zu erhalten und sich zeitgleich über den Aufkauf von Startups und etablierten Firmen ihre oligopolistische Stellung in der neuen Welt zu sichern, indem sie Dienstleistungen und Infrastrukturen anbieten, die eine Kontrolle des gesamten Forschungsprozess erlauben (siehe dazu z. B. Posada und Chen 2018). Open Access und der Wegfall von Subskriptionsgebühren erfordern aber auch die Entwicklung neuer Geschäfts- und Finanzierungsmodelle sowie neuer Publikationsangebote. In der Folge wollen wir vier solcher Veränderungen skizzieren, die unserer Ansicht nach für den Wandel des wissenschaftlichen Publikationssystems stehen. ${ }^{15}$

\footnotetext{
${ }^{14} \mathrm{http}: / / \mathrm{www}$. snf.ch/de/derSnf/forschungspolitische_positionen/open_access/Seiten/default.aspx.

${ }^{15}$ Für eine ausführliche Übersicht über Geschäftsmodelle für Open Access siehe Speicher et al. (2018).
} 
Open-Access-Publikationsgebühren Für diese Form der Finanzierung haben sich die Begriffe Article Processing Charges (APC), Book Processing Charges (BPC) und Book Chapter Processing Charges (BCPC) durchgesetzt. Bezahlt werden die Gebühren entweder von den AutorInnen selbst, von Forschungsförderern oder von den Institutionen, an denen die AutorInnen beschäftigt sind. Da viele Verlage, unabhängig davon ob es sich um eine Open-Access-Publikation handelt oder nicht, Gebühren für die Bearbeitung und Publikation von Texten fordern (für Lektorat, Farbabbildungen, Seitengebühren usw.), passt diese Form der Finanzierung in bereits vorhandene Geschäftsprozesse vieler Verlage. Darüber wie hoch Publikationsgebühren sein müssen oder welche Beträge angemessen sind, gibt es keinen Konsens. So differieren die Kosten zwischen verschiedenen Verlagen oder auch Zeitschriften um mehrere tausend Dollar. Außerdem ist zweifelhaft, ob APC-basiertes Open Access eine Lösung für das Kostenproblem der Wissenschaftskommunikation bieten kann. So kommt eine Studie von Universities UK zu dem Schluss, dass die durchschnittlichen Preise der APCs zwischen 2013 und 2016, die von englischen Universitäten bezahlt wurden, um $16 \%$ gestiegen sind (Universities UK 2017).

Gemeinschaftliche Finanzierung von Publikationen Eine Alternative zur Bezahlung von APCs ist die gemeinschaftliche Finanzierung von Publikationen. Bei diesem mit dem Crowdfunding vergleichbaren Modell werden die Publikationskosten freiwillig von Institutionen übernommen, die sich an der Finanzierung beteiligen. Dieses Modell findet sich häufig bei Non-Profit-Initiativen, wie bspw. Language Science Press oder Open Library of Humanities, die in der fachwissenschaftlichen Community verwurzelt sind.

Neue Universitätsverlage und Academic-led Presses Neben der Tatsache, dass die meisten großen Verlage in der Zwischenzeit Open-Access-Modelle für die Buchpublikationen anbieten, sind seit den letzten Jahren eine Reihe von neuen Universitätsverlagen entstanden (Bargheer und Pabst 2016). Zu nennen sind hier z. B. UCL Press am University College London, Lund University Press und der Universitätsverlag Göttingen. Aber auch Wissenschaftler betätigen sich als Herausgeber von Büchern und Zeitschriften. Zu diesen sogenannten Academic-led Presses gehören bspw. Open Humanities Press oder MayFly Books. Auch wenn sich diese Verlagsangebote im Hinblick auf die Größe und den Umfang der angebotenen Dienstleistungen stark unterscheiden, haben sie gemeinsam, dass sie nicht profitorientiert sind (Adema und Stone 2017). Finanziert werden die Publikationen meist mit Fördergeldern, Crowdfunding oder über Publikationsbeiträge.

Verbreitungsrechte Verfügen die Verlage über die exklusiven Verbreitungsrechte, können sie z. B. für eine weitere Publikation des Texts oder von Varianten des Texts von den Autoren Geld verlangen. Möglich ist das auch bei Texten, die Open Access erscheinen, wenn eine Lizenz gewählt wird, die eine kommerzielle Nutzung ausschließt, der Text aber in einer anderen Verlagspublikation erscheinen soll. Dann kann die Wiederverwendung 
als kommerzielle Nutzung betrachtet werden und die AutorInnen müssen den Verlag, der die Verbreitungsrechte hält, entschädigen. Viele neuere Open-Access-Verlage verzichten zugunsten einer einfachen Publikationsvereinbarung auf eine solche Rechteübertragung. Sollte sich der Verzicht auf die exklusive Übertragung der Verbreitungsrechte an die Verlage durchsetzen, wie das auch der Plan S und die nationale Open-Access-Strategie der Schweiz anstreben, werden der Monetarisierung der Texte durch die Verlage Grenzen gesetzt. Dadurch, dass die Verbreitungsrechte bei den Autoren bleiben, können Verlage für eine nochmalige Publikation der Texte keine Gebühren verlangen.

Der Verzicht auf die Übertragung ausschließlicher Verbreitungsrechte würde jedoch deutlich stärkere Veränderungen bewirken, als der Wegfall gelegentlicher Einnahmen durch die Zweitpublikation von einzelnen Artikeln. Denn ohne die exklusiven Verbreitungsrechte kann mit diesen nicht gehandelt werden. Wenn aber Medienkonzerne andere aufkaufen, Partnerschaften eingehen oder Teile ihres Portfolios verkaufen, geht es gerade um ebendiese Rechte. Blieben die Verbreitungsrechte bei den Autoren, könnte dies deutliche Auswirkungen auf die Dynamik und die fortschreitende Oligopolisierung des wissenschaftlichen Publikationsmarkts haben (siehe dazu Larivière et al. 2015). Ohne den Besitz der Verbreitungsrechte wären Verlage nicht mehr die Besitzer der wissenschaftlichen Literatur, sondern lediglich Dienstleister, die von den Autoren die Erlaubnis zum Publizieren eines Texts erhalten.

\subsection{Abschluss und Ausblick: Open Access in der Schweiz}

Abschließend bleibt festzuhalten, dass in der Schweiz konzertiert und dezidiert an der Erreichung von Open Access gearbeitet wird (siehe Hirschmann und Verdicchio 2017). Im Rahmen der nationalen Open-Access-Strategie laufen Verhandlungen zu Read-and-Publish-Verträgen, die Subskriptionszahlungen mit Gebühren für Open-Access-Veröffentlichungen kombinieren. Ziel der Verhandlungen sind außerdem Kostentransparenz (also der Verzicht auf Geheimhaltungsklauseln) und Kostenneutralität (siehe swissuniversities 2018). Die Strategie des Schweizerischen Nationalfonds ist klar und unterstützt auch explizit Open Access für Buchpublikationen. Einfluss auf die Entwicklung von Open Access in der Schweiz haben nicht zuletzt die wissenschaftlichen Akademien. Die Schweizerische Akademie der Geistes- und Sozialwissenschaften hat 2016 eine Open-Access-Strategie verabschiedet und fördert seitdem die Umstellung der von ihr geförderten Zeitschriften zu Open Access (SAGW 2016). Mit dem SBFI, swissuniversities, dem SNF und der SAGW bekennen sich wichtigsten Wissenschaftsorganisationen und Förderer in der Schweiz zur Forderung und Förderung von Open Access.

Auch an den Universitäten bewegt sich in Bezug auf Open Access einiges. Mit BOP Serials in Bern und HOPE in Zürich stehen bisher zwei Plattformen zur Publikation von Open-Access-Zeitschriften zur Verfügung, die das Hosting von Zeitschriften kostenlos oder kostengünstig anbieten. Weitere Angebote für das Zeitschriftenhosting und der Produktion von Büchern sind im Aufbau. Einige Verlage und Druckereien reagieren auf 
die veränderte Publikationslandschaft, indem sie Verlagsdienstleistungen anbieten, die für universitäre Publikationsdienste attraktiv sein können. Anstatt die Produktion eines Buches vom Manuskript bis zum Verkauf zu organisieren, werden modulare Angebote entwickelt, die einen Ausschnitt des Buchproduktionsprozess abdecken. Inwiefern kleine, alternative Publikationsmöglichkeiten wirklich ,disruptiv“ wirken wie das Adema und Stone (2017, S. 3) erwarten, muss sich allerdings erst noch zeigen. Viel eher scheint es, dass die Medienkonzerne, die die derzeitige Publikationslandschaft dominieren, weiterhin eine wichtige Rolle spielen. Hier bleibt zu hoffen, dass eine Abhängigkeit von wenigen Konzernen wie sie im Publikationswesen besteht, für die anderen Arbeitsbereiche von Open Science vermieden werden kann.

In absehbarer Zeit werden auch für kleinere Hochschulen Infrastrukturen für den grünen Weg zu Open Access zur Verfügung stehen. Dies entweder über Infrastrukturkollaborationen, wie bspw. die Zusammenarbeit zwischen der Berner Fachhochschule mit der Universität Bern, die Hochschulen in Luzern mit der ZHB Luzern oder über die sich im Aufbau befindliche Plattform SONAR, die von kleineren Hochschulen benutzt werden kann. Weitere Entwicklungen in Richtung Open Science werden höchstwahrscheinlich verstärkt zu Dienstleistungskooperationen zwischen verschiedenen Hochschulen, z. B. im Bereich des Forschungsdatenmanagement oder der Datenkuratierung, führen. Wenig verbreitet sind bisher Forschungsinformationssysteme, die für Open Science eine wichtige Rolle spielen können und eine öffentlich sichtbare Verknüpfung von Projekten, Personen, Publikationen und Forschungsdaten leisten. Die Systeme, die bisher in der Schweiz im Einsatz sind, werden außer in Luzern vor allem für interne Zwecke eingesetzt.

$\mathrm{Ob}$ die Schweiz das von der nationalen Strategie formulierte Ziel erreichen wird, muss abgewartet werden. Auch wenn die Open-Access-Rate im internationalen Vergleich gut aussieht, müssen noch erhebliche Anstrengungen unternommen werden, wenn bis 2024 alle öffentlich finanzierten Publikationen offen zugänglich sein sollen. Darüber hinaus kann man gespannt sein, ob und wie es in der Schweiz gelingen wird, den Wandel zu Open Science zu gestalten und dabei lokale und disziplinäre Diversität zu erhalten.

\section{Literatur}

Adema, J., \& Stone C. (2017). Changing publishing ecologies. A landscape study of New University Presses and Academic-Led Publishing. JISC. http://repository.jisc.ac.uk/id/eprint/6666. Zugegriffen: 16. Apr. 2019.

Arza, V., \& Fressoli, M. (2017). Systematizing benefits of open science practices. Information Services \& Use, 37(4), 463-474. https://doi.org/10.3233/isu-170861.

Bargheer, M., \& Pabst, J. (2016). Being small is not a fault': Making sense of the newer generation of German-Language University Presses. Learned Publishing, 29(1), 335-341. https://doi. org/10.1002/leap.1053.

Buranyi, S. (27. Juni 2017). Is the staggeringly profitable business of scientific publishing bad for science? The Guardian. https://www.theguardian.com/science/2017/jun/27/profitable-business-scientific-publishing-bad-for-science. Zugegriffen: 16. Apr. 2019. 
Cambridge Economic Policy Associates Ltd. (CEPA). (2017). Financial flows in Swiss publishing. https://doi.org/10.5281/zenodo.240896.

cAOlition S (2018). Plan S: Accelerating the transition to full and immediate open access to scientific publications. https://www.coalition-s.org/wp-content/uploads/Plan_S.pdf. Zugegriffen: 16. Apr. 2019.

Christen, M. (2017). Aufkommen von hybridem Open Access in der Schweiz am Beispiel der ETH Zürich. o-bib. Das offene Bibliotheksjournal, 4(3), 67-87. https://doi.org/10.5282/obib/2017h3s67-87.

Deppe, A., \& Beucke, D. (2017). Urspünge und Entwicklung von Open Access. In K. Söllner \& B. Mittermaier (Hrsg.), Praxishandbuch Open Access (S. 12-20). Berlin: De Gruyter. https://doi. org/10.5281/zenodo.802639.

European Commission. (2017). Providing researchers with the skills and competencies they need to practise open science. https://doi.org/10.2777/121253.

European Commission. (2018). Cost-benefit analysis for FAIR research data. https://publications. europa.eu/en/publication-detail/-/publication/d375368c-1a0a-11e9-8d04-01aa75ed71a1/language-en. Zugegriffen: 16. Apr. 2019.

European Commission. (2019). Open science monitor. https://ec.europa.eu/info/research-and-innovation/strategy/goals-research-and-innovation-policy/open-science/open-science-monitor/ trends-open-access-publications_en\#open-access-to-publications. Zugegriffen: 16. Apr. 2019.

Ferwerda, E., Snijder, R., Arpagaus, B., Graf, R., Krämer, D., \& Moser, E. (2018). OAPEN-CH Auswirkungen von Open Access auf wissenschaftliche Monographien in der Schweiz. Ein Projekt des Schweizerischen Nationalfonds. https://doi.org/10.5281/zenodo.1219172.

Gutknecht, C., Graf, R., Kissling, I., Krämer, D., Milzow, K., Perini, L., Würth, S., \& Zimmermann, T. (2016). SNSF monitoring report: Open access to publications 2013-2015. Swiss National Science Foundation. http://www.snf.ch/SiteCollectionDocuments/Monitoringbericht_ Open_Access_2015_d.pdf. Zugegriffen: 16. Apr. 2019.

Hagner, M. (23. Mai 2014). Gute Bücher benötigen Zeit und Papier. Neue Zürcher Zeitung. https:// www.nzz.ch/feuilleton/gute-buecher-benoetigen-zeit-und-papier-1.18307870. Zugegriffen: 19. Apr. 2019.

Hirschi, C. (19. Mai 2014). Der Schweizerische Nationalfonds und seine Open-Access-Strategie. Neue Zürcher Zeitung. https://www.nzz.ch/feuilleton/der-schweizerische-nationalfonds-und-seine-open-access-strategie-1.18304812. Zugegriffen: 19. Apr. 2019.

Hirschmann, B., \& Verdicchio, D. (2017). Open Access in der Schweiz. In K. Söllner \& B. Mittermaier (Hrsg.), Praxishandbuch Open Access (S. 215-222). Berlin: De Gruyter. https://doi. org/10.1515/9783110494068-025.

Larivière, V., Haustein, S., \& Mongeon, P. (2015). The oligopoly of academic publishers in the digital era. PLoS ONE, 10(6), e0127502. https://doi.org/10.1371/journal.pone.0127502.

Levin, N., \& Leonelli, S. (2017). How does one ,open" science? Questions of value in biological research. Science, Technology and Human Values, 42(2), 280-305. https://doi. org/10.1177/0162243916672071.

Manola, N., Rettberg, N., \& Manghi, P. (2015). OpenAIREplus project executive report. https:// doi.org/10.5281/zenodo.15464.

McKiernan, E. C. et al. (2016). Point of view: How open science helps researchers succeed. ELife. https://doi.org/10.7554/elife.16800.

Merton, R. K. (1973). The normative structure of science. In R. K. Merton (Hrsg.), The sociology of science: Theoretical and empirical investigations (S. 267-278). Chicago: University of Chicago Press.

Morrison, H. (2012). Freedom for scholarship in the internet age. http://summit.sfu.ca/item/12537. Zugegriffen: 16. Apr. 2019. 
Morrison, H. (2014). Economics of scholarly communication in transition. First Monday. https:// doi.org/10.5210/fm.v18i6.4370.

Posada, A., \& Chen, G. (2018). Inequality in knowledge production: The integration of academic infrastructure by big publishers. In L. Chan \& P. Mouner (Hrsg.), ELPUB 2018. https://doi. org/10.4000/proceedings.elpub.2018.30.

Reichmann, W. (2017). Open Science zwischen sozialen Strukturen und Wissenskulturen. TATuP Zeitschrift für Technikfolgenabschätzung in Theorie und Praxis, 26, 43-48. https://doi. org/10.14512/tatup.26.1-2.43.

Sample, I. (24. April 2012). Harvard University says it can't afford journal publishers' prices. The Guardian. https://www.theguardian.com/science/2012/apr/24/harvard-university-journal-publishers-prices. Zugegriffen: 16. Apr. 2019.

Savary, G. (2014). Interpellation 14.3215: Open Access. Eine Bedrohung für das Verlagswesen? Bundesversammlung - Das Schweizer Parlament 20. März 2014. https://www.parlament.ch/de/ ratsbetrieb/suche-curia-vista/geschaeft?Affairld=20143215. Zugegriffen: 19. Apr. 2019.

Schweizerische Akademie der Geistes- und Sozialwissenschaften (SAGW). (2016). Open-Access-Strategie der Schweizerischen Akademie der Geistes- und Sozialwissenschaften. https:// sagw.ch/fileadmin/user_upload/Open-Access-Strategie_4_.pdf. Zugegriffen: 16. Apr. 2019.

Speicher, L., Armando, 1., Bargheer, M., Eve, M. P., Fund, S., Leão, D., Mosterd, M., Pinter, F., \& Souyioultzoglou, I. (2018). OPERAS open access business models white paper. https://doi. org/10.5281/zenodo.1323708.

Staatssekretariat für Bildung, Forschung und Innovation (SBFI). (2015). Brief an die Präsidentin Swissuniversities vom 04.12.2018, Betr. Nationale Strategie Open Access. https://www.swissuniversities.ch/fileadmin/swissuniversities/Dokumente/Hochschulpolitik/Open_Access/151204_ Brief_SBFI_OA_nationale_Strategie_Swissuniversities_sign.pdf. Zugegriffen: 16. Apr. 2019.

Swissuniversities. (2018). Factsheet zur Verhandlungsstrategie. https://www.swissuniversities.ch/ fileadmin/swissuniversities/Dokumente/Hochschulpolitik/Open_Access/180315_Factsheets_ Verhandlungsstrategie_D.pdf. Zugegriffen: 16. Apr. 2019.

Swissuniversities, \& Schweizerischer Nationalfonds. (2016). Nationale Open-Access-Strategie für die Schweiz. https://www.swissuniversities.ch/fileadmin/swissuniversities/Dokumente/Hochschulpolitik/Open_Access/Open_Access_strategy_final_DE.pdf. Zugegriffen: 16. Apr. 2019.

Tulney, M. (2018). Plan S - Erste Implementierungsrichtlinien für Open-Access-Auflagen. TIB-Blog. https://blogs.tib.eu/wp/tib/2018/11/27/plan-s-erste-implementierungsrichtlinien-fuer-open-access-auflagen. Zugegriffen: 16. Apr. 2019.

Universities UK. (2017) Monitoring the transition to open access. https://www.universitiesuk. ac.uk/policy-and-analysis/reports/Documents/2017/monitoring-transition-open-access-2017. pdf. Zugegriffen: 16. Apr. 2019.

Weik, R. (7. Mai 2014). „Ein Schlag ins Gesicht“. Tagblatt. https://www.tagblatt.ch/ostschweiz/ ein-schlag-ins-gesicht-ld.660820. Zugegriffen: 19. Apr. 2019.

Verdicchio Dirk (Dr. phil./dirk.verdicchio@ub.unibe.ch) Ko-Leiter des Bereichs Digitale Dienste und Open Science an der Universitätsbibliothek Bern. Verantwortlich für Dienstleistungen und Infrastrukturen für Open Science (Open Access, Forschungsdaten, Forschungsinformationen, Publikationsdienste). Dozent für Wissenschaftskommunikation am Studiengang Bibliotheks- und Informationswissenschaft, Universität Zürich. Promotion in Soziologie an der Universität Basel um Thema Wissenschaftskommunikation in Filmen. 
Kissling-Näf, Ingrid (Prof. Dr./ingrid.kissling@bfh.ch) Direktorin Departement Wirtschaft der Berner Fachhochschule.

Forschungserfahrung als Assistenzprofessorin in Ressourcenökonomie an der ETHZ und langjährige Führungserfahrung unter anderem beim Schweizerischen Nationalfonds Abteilung Geistesund Sozialwissenschaften, bei der Förderagentur für Innovation KTI sowie bei der Akademie der Naturwissenschaften Schweiz . Während zwei Jahren tätig als Beraterin in der Privatwirtschaft bei der Firma Ernst Basler \& Partner AG. Sie ist zudem UNICEF-Delegierte und Stadträtin in Bern.

Open Access Dieses Kapitel wird unter der Creative Commons Namensnennung 4.0 International Lizenz (http://creativecommons.org/licenses/by/4.0/deed.de) veröffentlicht, welche die Nutzung, Vervielfältigung, Bearbeitung, Verbreitung und Wiedergabe in jeglichem Medium und Format erlaubt, sofern Sie den/die ursprünglichen Autor(en) und die Quelle ordnungsgemäß nennen, einen Link zur Creative Commons Lizenz beifügen und angeben, ob Änderungen vorgenommen wurden.

Die in diesem Kapitel enthaltenen Bilder und sonstiges Drittmaterial unterliegen ebenfalls der genannten Creative Commons Lizenz, sofern sich aus der Abbildungslegende nichts anderes ergibt. Sofern das betreffende Material nicht unter der genannten Creative Commons Lizenz steht und die betreffende Handlung nicht nach gesetzlichen Vorschriften erlaubt ist, ist für die oben aufgeführten Weiterverwendungen des Materials die Einwilligung des jeweiligen Rechteinhabers einzuholen.

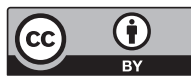

